\section{Cherry-flavoured electronic cigarettes expose users to the inhalation irritant, benzaldehyde}

\begin{abstract}
Many non-cigarette tobacco products, including e-cigarettes, contain various flavourings, such as fruit flavours. Although many flavourings used in e-cigarettes are generally recognised as safe when used in food products, concerns have been raised about the potential inhalation toxicity of these chemicals. Benzaldehyde, which is a key ingredient in natural fruit flavours, has been shown to cause irritation of respiratory airways in animal and occupational exposure studies. Given the potential inhalation toxicity of this compound, we measured benzaldehyde in aerosol generated in a laboratory setting from flavoured ecigarettes purchased online and detected benzaldehyde in 108 out of 145 products. The highest levels of benzaldehyde were detected in cherry-flavoured products. The benzaldehyde doses inhaled with 30 puffs from flavoured e-cigarettes were often higher than doses inhaled from a conventional cigarette. Levels in cherry-flavoured products were $>1000$ times lower than doses inhaled in the workplace. While e-cigarettes seem to be a promising harm reduction tool for smokers, findings indicate that using these products could result in repeated inhalation of benzaldehyde, with long-term users risking regular exposure to the substance. Given the uncertainty surrounding adverse health effects stemming from long-term inhalation of flavouring ingredients such as benzaldehyde, clinicians need to be aware of this emerging risk and ask their patients about use of flavoured e-cigarettes.
\end{abstract}

Current estimates show that $12.6 \%$ of US adults report ever use of electronic cigarettes (e-cigarettes), a number that continues to rise. ${ }^{1}$ Recent studies confirm that e-cigarettes presumably pose lower health risks to the user due to the disparity in toxic compounds found in e-cigarettes relative to tobacco cigarettes. ${ }^{2}$ While the 2009 Family Smoking Prevention and Tobacco Control Act gave rise to a US ban on added, non-menthol flavourings in cigarettes, ${ }^{3}$ currently, non-cigarette tobacco products, including e-cigarettes, are permitted to have added flavourings (eg, candy, fruit). Although many flavourings used in e-cigarettes are generally recognised as safe when used in food products, concerns have been raised about the potential inhalation toxicity of these chemicals. ${ }^{4}$ The potential effects flavour compounds may have on the respiratory health of e-cigarette users are unclear. A recent report by Barrington-Trimis et $a l^{4}$ outlined the need for research on flavour additives used in e-cigarettes, noting the lack of safety data on long-term health effects from inhaling flavouring chemicals.

One such flavouring compound, benzaldehyde, is an aromatic aldehyde used in food and cosmetics. ${ }^{5}$ Although data suggest little to no toxicity from oral and dermal exposure to benzaldehyde, occupational exposure has been linked to irritation of the eyes and mucous membranes of the respiratory passages. ${ }^{5}$ Given the potential inhalation toxicity of benzaldehyde to e-cigarette users, we examined benzaldehyde levels generated from flavoured ecigarettes. We hypothesised that aerosol generated from flavoured e-cigarettes would contain significant amounts of benzaldehyde.

\section{METHODS}

We measured benzaldehyde in aerosol generated from an e-cigarette refilled with 145 flavoured nicotine-containing solutions purchased from international online retailers. The solutions were classified into eight flavour groups according to labelled characteristics: berry/tropical fruit $(n=40)$, tobacco $(n=37)$, alcohol related/ drink $(n=15)$, chocolate/sweet flavour $(n=11)$, coffee/tea $(n=11), \operatorname{mint} /$ menthol $(n=10)$, cherry $(n=10)$, and other, nonidentifiable flavour varieties (eg, 'Indian Summer', 'Cosmopolitan') ( $\mathrm{n}=11)$. A refillable eGo e-cigarette (heater resistance $2.4 \mathrm{ohm}$, battery $900 \mathrm{mAh}, 3.4 \mathrm{~V}$ ) was used for testing. Aerosol was generated using an automatic smoking simulator following protocols used in previous studies (inhalation time $1.8 \mathrm{~s}$, puff volume $70 \mathrm{~mL}$, interval between puffs $17 \mathrm{~s}) .^{6}$ For each experiment, 30 puffs were taken from each e-cigarette in two series of 15 puffs, with a 5 min interval between series. Benzaldehyde from aerosol was trapped on sorbent tubes, extracted and analysed using a high-performance liquid chromatography method as recommended by the US Environmental Protection Agency. ${ }^{7}$ The lower limit of quantitation (LLOQ) was $0.025 \mu \mathrm{g} / 30$ puffs. A Kruskal-Wallis test was performed to assess differences in the distributions of benzaldehyde levels according to flavour grouping. For each product tested, we calculated a daily inhaled dose of benzaldehyde, assuming that an experienced e-cigarette user puffs on an e-cigarette 163 times a day. ${ }^{8}$ We compared the inhaled dose using 30 puffs from a flavoured e-cigarette with an inhaled dose from a single tobacco cigarette (0.5$4.5 \mu \mathrm{g} /$ cigarette). ${ }^{9}$ We also compared the inhaled dose from flavoured e-cigarettes with a hypothetical dose inhaled by healthy workers who would be exposed during an $8 \mathrm{~h}$ work shift to the permissible exposure limit (PEL) of benzaldehyde as defined by the US Workplace Environmental Exposure Level Guides ${ }^{10}$ (90.5 $\mathrm{mg}=$ inhalation rate $1.34 \mathrm{~m}^{3} / \mathrm{h} \times 8$ $\left.\mathrm{h} \times 8.7 \mathrm{mg} / \mathrm{m}^{3} \mathrm{PEL}\right)$.

\section{RESULTS}

Benzaldehyde was detected in 108 out of 145 examined products (levels above LLOQ). The highest yields of benzaldehyde were observed in cherry-flavoured products (5.129-141.2 $\mu \mathrm{g} / 30 \mathrm{puffs})$. Benzaldehyde yields in aerosol generated from cherry-flavoured nicotine solutions were significantly higher than in other products $\quad\left(\chi^{2}(7)=43.70, \quad \mathrm{p}<0.0001\right.$; Kruskal-Wallis test). Benzaldehyde yields in non-cherry-flavoured products were within the range of $0.025-10.27 \mu \mathrm{g} /$ 30 puffs (table 1). The benzaldehyde doses inhaled using 30 puffs from flavoured e-cigarettes were often higher than doses inhaled from a conventional cigarette. The estimated median daily inhaled dose of benzaldehyde from cherryflavoured e-cigarettes was $70.3 \mu \mathrm{g}$, which would be $>1000$ times lower than the PEL dose for benzaldehyde concentrations in the workplace (figure 1).

\section{DISCUSSION}

By examining benzaldehyde levels in aerosols generated from a wide range of flavoured nicotine solutions, our study demonstrated that elevated levels of benzaldehyde exist in cherry-flavoured products. Users of cherry-flavoured products may inhale significantly higher doses of benzaldehyde compared with users of other flavoured products. Although this study has several limitations, including an arbitrarily selected testing protocol that may not reflect actual product use, this study points out a potential risk associated with using flavoured e-cigarettes.

As e-cigarette popularity increases, respiratory clinicians need to be aware of potential risks that may result from use. Although e-cigarettes may be a promising harm reduction tool for smokers, findings indicate that using these products could result in repeated inhalation of benzaldehyde, with long-term users risking regular exposure to the substance. Given the uncertainty surrounding adverse health effects stemming from long-term inhalation of flavouring ingredients such as benzaldehyde, patients should be asked 
Table 1 Yields of benzaldehyde in aerosol generated from flavoured e-cigarettes $(n=145)$

\begin{tabular}{|c|c|c|c|c|}
\hline Flavour & Number of tested products & $\begin{array}{l}\text { Proportion of products with } \\
\text { positive benzaldehyde } \\
\text { ( }>\text { greater than } \\
\text { limit of quantitation) (\%) }\end{array}$ & $\begin{array}{l}\text { Median benzaldehyde } \\
\text { yield ( } \mu \mathrm{g} / 30 \text { puffs) }\end{array}$ & $\begin{array}{l}\text { Range of benzaldehyde } \\
\text { yields ( } \mu \mathrm{g} / 30 \text { puffs) }\end{array}$ \\
\hline Fruit (other than cherry) & 40 & 62.5 & 0.050 & BLQ-2.041 \\
\hline Tobacco & 37 & 78.4 & 0.219 & BLQ-2.093 \\
\hline Alcohol related or drink & 15 & 73.3 & 0.118 & BLQ-4.499 \\
\hline Coffee or tea & 11 & 81.8 & 0.143 & BLQ-3.010 \\
\hline Chocolate or sweet flavour & 11 & 90.9 & 0.130 & BLQ-5.327 \\
\hline Mint or menthol & 10 & 40.0 & 0.025 & BLQ—0.237 \\
\hline Cherry & 10 & 100 & 12.93 & 5.129-141.2 \\
\hline Other & 11 & 90.9 & 0.321 & BLQ—10.27 \\
\hline
\end{tabular}

BLQ, below limit of quantitation ( $0.025 \mu \mathrm{g} / 30$ puffs).

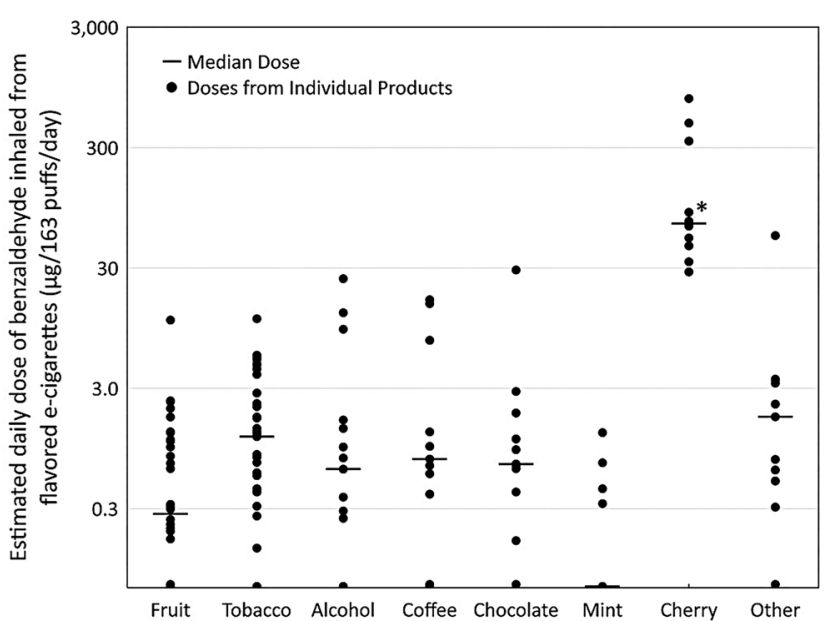

Figure 1 Estimated daily doses of benzaldehyde resulting from inhaling flavoured e-cigarette aerosol (163 puffs per day $\left.{ }^{10}\right)(n=145)$.

about use of flavoured products, particularly patients with respiratory diseases. Leon Kosmider, ${ }^{1,2}$ Andrzej Sobczak, ${ }^{1,2}$
Adam Prokopowicz, ${ }^{1}$ Jolanta Kurek, ${ }^{1}$
Marzena Zaciera, ${ }^{1}$ Jakub Knysak, ${ }^{2}{ }^{1}{ }^{3}$
Danielle Smith, ${ }^{3}$ Maciej L Goniewicz ${ }^{3}$

${ }^{1}$ Institute of Occupational Medicine and Environmental Health, Sosnowiec, Poland

${ }^{2}$ Department of General and Inorganic Chemistry, Medical University of Silesia, Katowice, Poland

${ }^{3}$ Department of Health Behavior, Roswell Park Cancer Institute, Buffalo, New York, USA

Correspondence to Dr Maciej L Goniewicz, Department of Health Behavior, Roswell Park Cancer Institute, Elm \& Carlton Streets/Carlton House A320, Buffalo, NY 14263, USA; maciej.goniewicz@ roswellpark.org

Contributors Conception and design: $\mathrm{LK}, \mathrm{AS}$ and MLG. Analysis and interpretation: AP, JK, MZ, JK, DS and MLG. Drafting the manuscript for important intellectual content: AS, DS and MLG.

Funding This research was supported by the Medical University of Silesia (grants KNW-2-007/D/4/N and KNW-2-016/D/5/K), Institute of Occupational and Environmental Health (grant ZSiTG9) and by NIDA/NIH and FDA Center for Tobacco Products (CTP) (grant 3R01DA037446)

Competing interests $L K, A S, A P, J K$ and $M Z$ are employees of the Institute of Occupational Medicine and Environmental Health. One of the institute's objectives is outsourcing for the industrial sector, including manufacturers of e-cigarettes. However, this has no influence on studies design, conducting studies, manuscript preparation, statistical analysis or other relevant scientific work conducted in the Institute of Occupational Medicine and Environmental Health. AS accepted personal fees from the eSmoking Institute in Poznan, Poland, and non-financial support from Chic Group LTD, a manufacturer of electronic cigarettes in Poland, outside of the submitted work. In 2011, MLG received the Global Research Award for Nicotine Dependence funded by Pfizer, a manufacturer of stop smoking medications.

Provenance and peer review Not commissioned; externally peer reviewed.

Data sharing statement Data could be made corresponding author.

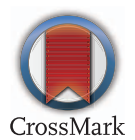
available to qualified researchers by request to the
To cite Kosmider L, Sobczak A, Prokopowicz A, et al. Thorax 2016:71:376-377.

Received 4 October 2015

Revised 18 November 2015

Accepted 24 November 2015

Published Online First 28 January 2016

Thorax 2016;71:376-377.

doi:10.1136/thoraxjn-2015-207895

\section{REFERENCES}

1 Schoenborn CA, Gindi RM. Electronic cigarette use among adults: United States. NCHS Data Brief, no 217. 2014. Hyattsville, MD: National Center for Health Statistics, 2015.

2 Goniewicz ML, Knysak J, Gawron M, et al. Levels of selected carcinogens and toxicants in vapour from electronic cigarettes. Tob Control 2014;23: 133-9.

3 Overview of the Family Smoking Prevention and Tobacco Control Act. June 2009. Retrieved from http://www.fda.gov/downloads/TobaccoProducts/ GuidanceComplianceRegulatorylnformation/ UCM336940.pdf

4 Barrington-Trimis JL, Samet JM, McConnell R. Flavorings in electronic cigarettes: an unrecognized respiratory health hazard? JAMA 2014;312: 2493-4.

5 Benzaldehyde [MAK Value Documentation, 2002]. The MAK Collection for Occupational Health and Safety. Wiley-VCH Verlag GmbH \& Co. KGaA, 2012:14-36.

6 Kosmider L, Sobczak A, Fik M, et al. Carbonyl compounds in electronic cigarette vapors: effects of nicotine solvent and battery output voltage. Nicotine Tob Res 2014;16:1319-26.

7 US Environmental Protection Agency (US EPA). Compendium of methods for the determination of toxic organic compounds in ambient air. Method TO-11A. Cincinnati, OH: US Environmental Protection Agency, 1999. Retrieved from http://www.epa.gov/ ttnamti1/files/ambient/airtox/to-11 ar.pdf

8 Dautzenberg B, Bricard D. Real-time characterization of e-cigarettes use: the 1 million puffs study. J Addict Res Ther 2015:6:2.

9 Pang X, Lewis AC. Carbonyl compounds in gas and particle phases of mainstream cigarette smoke. Sci Total Environ 2011;409:5000-9.

10 American Industrial Hygiene Association. WEEL Values. American Industrial Hygiene Association. 2011. https://www.aiha.org/get-involved/ AIHAGuidelineFoundation/WEELs/Documents/ 2011WEELValues.pdf (accessed 25 Aug 2015). 